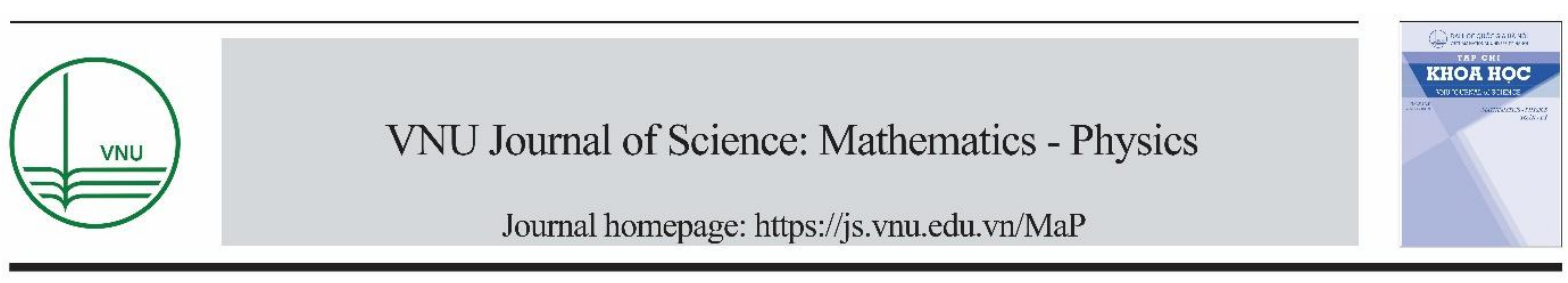

Original Article

\title{
Influence of Sr Doping on Magnetic and Magnetocaloric Properties of $\mathrm{Nd}_{0.6} \mathrm{Sr}_{0.4} \mathrm{MnO}_{3}$
}

\author{
Ho Thi Anh, Nguyen Ngoc Huyen, Pham Duc Thang* \\ Faculty of Engineering Physics and Nanotechnology, VNU University of Engineering and Technology, \\ 144 Xuan Thuy, Cau Giay, Hanoi, Vietnam
}

Received 11 October 2019

Revised 02 December 2019; Accepted 30 December 2019

\begin{abstract}
Nd}_{0.6} \mathrm{Sr}_{0.4} \mathrm{MnO}_{3}$ sample was fabricated by a solid-state reaction method and its magnetic, magnetocaloric properties were investigated. The Curie temperature, $T_{\mathrm{C}}$, at which a ferromagneticparamagnetic transition occurred was found to be of about $270 \mathrm{~K}$. An analysis using the Banejee's criterion of the experiment results for magnetization as a function of temperature and magnetic field and the universal curves of the normalized entropy change versus reduced temperature indicated that the sample undergo the second-order magnetic phase transition. Furthermore, the maximum magnetic entropy change that occurred near $T_{\mathrm{C}}$, measured at a magnetic field span of $50 \mathrm{kOe}$ was found to be of about $6.0 \mathrm{~J} / \mathrm{kg} . \mathrm{K}$, corresponding to a relative cooling power of $250 \mathrm{~J} / \mathrm{kg}$. These values are comparable to those of other manganites.
\end{abstract}

Keywords: Perovskite manganites; Magnetocaloric effect; Universal entropy

\section{Introduction}

Hole-doped perovskite manganites with a chemical formula of $\mathrm{R}_{1-\mathrm{x}} \mathrm{A}_{\mathrm{x}} \mathrm{MnO}_{3}(\mathrm{R}=\mathrm{La}, \mathrm{Nd}, \mathrm{Pr} ; \mathrm{A}=$ $\mathrm{Ca}, \mathrm{Ba}, \mathrm{Sr}$ ) have received a lot of attention due to their intriguing physical properties, and their applicability in the magnetic refrigeration technology based upon the magnetocaloric effect (MCE) [14]. This effect is associated with the temperature change of the suitable magnetic material under an applied magnetic field. Particularly, if the magnetic field is applied adiabatically, the temperature of the materials increases, and if the magnetic field is removed, the temperature decreases. Additionally, this effect is often expected to get maximum value at magnetic phase transition. It is well known that the

\footnotetext{
* Corresponding author.

Email address: thangducpham@yahoo.com
}

https//doi.org/ 10.25073/2588-1124/vnumap.4403 
properties of perovskite manganites is directly related to ferromagnetic (FM) or anti-ferromagnetic (AFM) ordering, charge ordering and orbital ordering [5-7]. The strength of FM and AFM interactions is associated with the double-exchange pair of $\mathrm{Mn}^{3+}-\mathrm{Mn}^{4+}$ and super-exchange pair of $\mathrm{Mn}^{3+}-\mathrm{Mn}^{3+}$ and $\mathrm{Mn}^{4+}-\mathrm{Mn}^{4+}$, respectively, depends on $\mathrm{Mn}^{3+}$ and $\mathrm{Mn}^{4+}$ concentrations and the structure parameters. The FM interaction becomes strongest when the concentration ratio of $\mathrm{Mn}^{3+} / \mathrm{Mn}^{4+}$ is about $7 / 3$, corresponding to an A-doping content $\mathrm{x} \approx 0.3$. Accordingly, the giant MCE and colossal magnetoresistance effects are usually obtained in the region $0.2 \leq \mathrm{x} \leq 0.4$ for most $\mathrm{R}_{1-\mathrm{x}} \mathrm{A}_{\mathrm{x}} \mathrm{MnO}_{3}$ systems [8].

The strength of double-exchange interactions in Nd-based manganites is usually weaker in comparation with La-based manganites, their magnetic properites are more interesting and complicated due to a larger lattice distortion would happen for smaller $\mathrm{Nd}$ ion. It is well known that the parent compound $\mathrm{NdMnO}_{3}$ is an insulating antiferromagnet with $T_{\mathrm{N}} \approx 78 \mathrm{~K}$ [9], with a small partial substitution $0<x<0.1$ of $\mathrm{Sr}$ for $\mathrm{Mn}$, the system becomes ferromagnetic but still insulating [1] while a ferromagnetic metallic phase appears in the region $0.2 \leq x \leq 0.4$. Venkatesh et. al [10] showed that a tricritical point was observed in the $\mathrm{Nd}_{1-\mathrm{x}} \mathrm{Sr}_{\mathrm{x}} \mathrm{MnO}_{3}(x=0.3,0.33$, and 0.4) at $x=0.33$ which separates the first-order transition in compound with $x=0.3$ and second-order transition in compound with $x=0.4$. Additionally, the complicated behavior at low fields in which the order of the transition could not be fixed and a second-order-like behavior at high fields was observed in $\mathrm{Nd}_{0.7} \mathrm{Sr}_{0.3} \mathrm{MnO}_{3}$ [11]. The complex ferromagnetic state in this compound due to a competition between the double exchange mechanism and correlations arising from coupled spin and lattice degrees of freedom. The MCE in this compound was also studied in two ways indirectly by estimating the isothermal change in entropy, $\Delta \mathrm{S}_{\mathrm{m}}$, and directly by measuring the field indiced adiabatic temperature change. However, up to now, the effect of Sr doping on magnetic and MC properties in $\mathrm{Nd}_{0.6} \mathrm{Sr}_{0.4} \mathrm{MnO}_{3}$ have been studied not much. To get more insight into these problems, we prepared $\mathrm{Nd}_{0.6} \mathrm{Sr}_{0.4} \mathrm{MnO}_{3}$ samples and carried out the magnetization measurements versus temperature and magnetic field. By combining the Banejee's criterion and the universal curves of the normalized entropy change versus reduced temperature, we assessed the magnetic order existing in this compound. We also studied the MCE by estimating $\Delta \mathrm{S}_{\mathrm{m}}$ from the magnetization data in different applied magnetic field.

\section{Experimental details}

Polycrystalline $\mathrm{Nd}_{0.6} \mathrm{Sr}_{0.4} \mathrm{MnO}_{3}$ was prepared by a conventional solid-state reaction. High-purity (99.9\%) powdered precursors of $\mathrm{Nd}_{2} \mathrm{O}_{3}, \mathrm{SrCO}_{3}$ and $\mathrm{MnO}_{2}$ with a specific composition, were well mixed, carefully ground, and then annealed at $900^{\circ} \mathrm{C}$ in air for $12 \mathrm{~h}$. The annealed samples were re-ground, pressed into pellets and calcinated at $1100^{\circ} \mathrm{C}$ in air for $24 \mathrm{~h}$. This process was done for serval times and finally the pellets were sintered in air at $1300^{\circ} \mathrm{C}$ for $10 \mathrm{~h}$. The single phase in an orthorhombic structure of the final sample was checked by an X-ray diffractometer using $\mathrm{Cu}-\mathrm{K} \alpha$ radiation over the $2 \Theta$ range of $20 \div 70^{\circ}$. The dependence of magnetization on temperature and applied magnetic field was measured by using a superconducting quantum interference device up to $50 \mathrm{kOe}$ in the temperature range of $5 \div 300 \mathrm{~K}$.

\section{Results and discussion}

Figure 1(a) shows temperature dependences of zero-field-cooled (ZFC) and field-cooled (FC) magnetization, $M_{Z F C / F C}(T)$, at such a small $H$ as 100 Oe for $\mathrm{Nd}_{0.6} \mathrm{Sr}_{0.4} \mathrm{MnO}_{3}$ sample. For the $M_{Z F C}(T)$ curve, $M$ increases with the increase of $T$, and reaches a maximum at $250 \mathrm{~K}$. This phenomenon is explained due to the existence of FM/AFM clusters and/or magnetic inhomogeneity [1]. Munoz et al. [9] have indicated the coexistence of FM and AFM interactions in polycrystalline $\mathrm{NdMnO}_{3}$. 
Additionally, with further increasing of temperature, above $250 \mathrm{~K}$, the magnetization was rapidly decreased due to the FM-PM phase transition, where magnetic moments became disorder under the impact of thermal energy. The Curie temperature, $T_{\mathrm{C}}$, determined from the minima of the first derivative of $M_{Z F C}(T) / \mathrm{d} T$ versus $T$ curve (not shown here) is about $270 \mathrm{~K}$. Similar behavior of $M_{Z F C}(T)$ and $T_{\mathrm{C}}$ values were found in the previous works $[1,10,12]$. Additionally, it is notable that the large difference between $M_{Z F C}(T)$ and $M_{F C}(T)$ was observed. In figure 1(b), this difference, $\Delta \mathrm{M}$, is plotted with respect to $T$ for this sample at $H=100 \mathrm{Oe}$. It is well known that $\Delta \mathrm{M}$ is small and independent on temperature for good ferromagnets. The large $\Delta \mathrm{M}$ implies a FM order and a rather high coercivity comparing to the applied field below $T_{\mathrm{C}}$. When applied field is smaller than the magnetic anisotropy which locked the magnetic moment of the $\mathrm{Mn}$ ions in the random direction, $\Delta \mathrm{M}$ is becoms larger. Moreover, the magnetic anisotropy was strong at temperature below $T_{\mathrm{C}}, \Delta \mathrm{M}$ increased as $T$ decreased. This behavior is clearly seen in Fig. 1(b). This result indicates that our sample was highly anisotropic and the applied field of 100 Oe was not strong enough to align the magnetic moments, resulting in a larger $\Delta \mathrm{M}$ at low $T$.
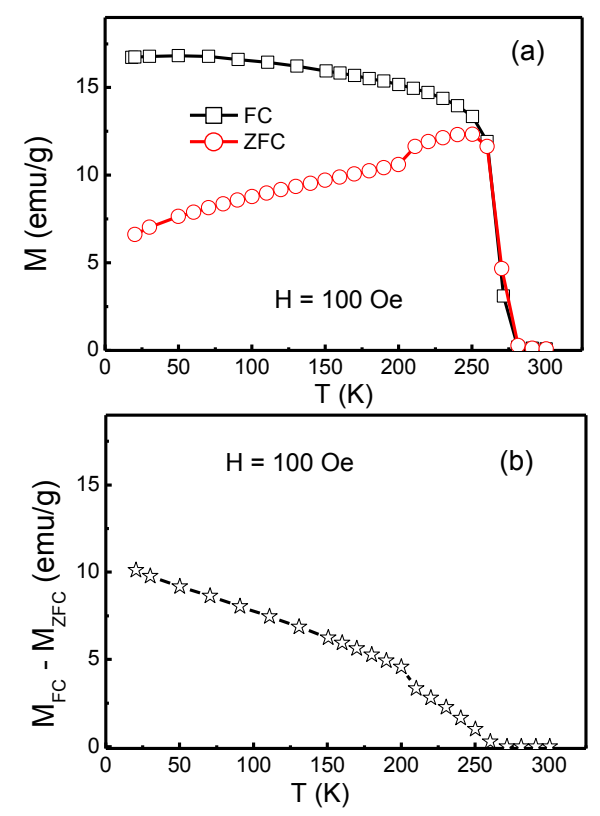

Figure 1. (a) $M_{\mathrm{ZFC}}(T)$ and $M_{\mathrm{FC}}(T)$ curves for $\mathrm{Nd}_{0.6} \mathrm{Sr}_{0.4} \mathrm{MnO}_{3}$ measured at $H=100$ Oe.

(b) $M_{\mathrm{FC}}-M_{\mathrm{ZFC}}$ with respect to $T$ for $\mathrm{Nd}_{0.6} \mathrm{Sr}_{0.4} \mathrm{MnO}_{3}$.

To understand the Sr-doping influence on magnetic and magnetocaloric properties of $\mathrm{Nd}_{0.6} \mathrm{Sr}_{0.4} \mathrm{MnO}_{3}$, series of isothermal magnetization curves, $M(H)$, were recorded at temperatures around $T_{\mathrm{C}}$ in the interval of $2 \mathrm{~K}$. Figure 2(a) shows the typical curves of $M(H)$ data for this sample. Clearly, like $M(T)$ data, at a given $H, M$ decreased with increasing temperature. In the FM-PM phase transition region, $M$ increased with $H$ increasing up to a magnetic field as large as $50 \mathrm{kOe}$. This probably is due to the coexistence of the FM and PM phase and magnetic inhomogeneities. This behavior was also observed for other manganite compounds [1, 13-16]. As temperature increased, the nonlinear $M(H)$ curves in FM region become linear that is the characteristic property of the PM state. The nature of FM-PM phase transition can be clearly observed in the Arrott plots [17], the $M^{2}$ dependence of $H / M$ curves are shown in Fig. 2(b). At high $H$ values, the plots near $T_{C}$ exhibit series of parallel straight lines, the straight line at $T=T_{\mathrm{C}}$ did not pass through the origin of ordinates. According to the mean-field theory applied for the long-range FM order, the existence of short-range FM order in our sample can be suggested. 
In order to check the nature of magnetic transition we have used the Banerjee's criterion [18]. By plotting $M^{2}$ vs. $H / M$ in the magnetic phase transition region, the slope of the resulting curves indicates whether a magnetic transition is of first or second order. From a thermodynamic point of view, one can deduce following, if some of the $M^{2}$ vs. $H / M$ curves near $T_{\mathrm{C}}$ have negative slope, the magnetic transition in the sample is a first-order magnetic transition. Otherwise, the entire slope is positive, the magnetic transition is a second-order magnetic transition (SOMT). The result shown in Fig. 2(b) indicates that the slope of the Arrott-plot curves near $T_{\mathrm{C}}$ is positive, this means that the existence of SOMT in our sample can be considered according to the Banerjee's criterion.
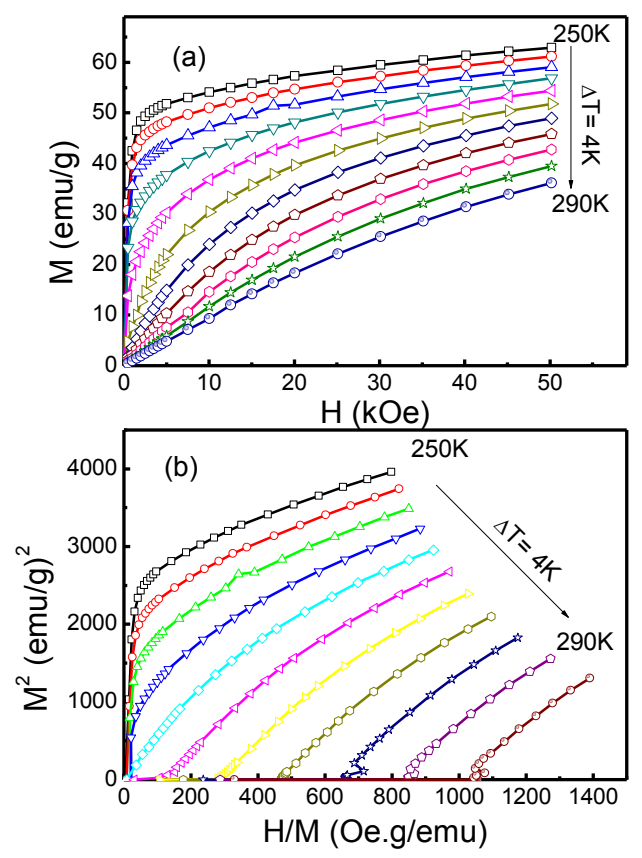

Figure 2. (a)-(b) $M(H)$ curves and (c)-(d) Arrott plots $\left(M^{2}\right.$ vs. $\left.H / M\right)$ for $\mathrm{Nd}_{0.6} \mathrm{Sr}_{0.4} \mathrm{MnO}_{3}$.

To evaluate the MCE in the sample, we assessed the magnetic entropy change $\Delta S_{\mathrm{m}}$, which can be calculated by using Maxwell's relation in the $H$ range of 0 to $H_{\max }$ [19]

$$
S_{m}(T, H)=-\int_{0}^{H \max }\left(\frac{\partial M}{\partial T}\right)_{H} d H
$$

Obviously, the slope of $\left(\frac{\partial M}{\partial T}\right)_{H}$ contribute the value of $\Delta S_{\mathrm{m}}$, indicating that an abrupt magnetic transition leading to large $\Delta S_{\mathrm{m}}$ and this value reach maximum around $T_{\mathrm{C}}$ where magnetization decays most rapidly[20]. For magnetization measurements with small, discrete field and temperature intervals, $\Delta S_{\mathrm{m}}$ could be numerically calculated using the following equation

$$
\left|S_{m}\right|=\sum \frac{M_{i}-M_{i+1}}{T_{i}-T_{i+1}} H_{i}
$$

Where $M_{\mathrm{i}}$ and $M_{\mathrm{i}+1}$ are magnetization values measured in a magnetic field $H$ at temperature $T_{\mathrm{i}}$ and $T_{\mathrm{i}+1}$, respectively. Figure 3(a) show the dependence of $-\Delta S_{\mathrm{m}}$ on temperature, $-\Delta S_{\mathrm{m}}(T)$, for different field variations at interval of $5 \mathrm{kOe}$. Clearly, at a specific temperature, $-\Delta S_{\mathrm{m}}$ increases with increasing $\Delta H$ from $5 \mathrm{kOe}$ to $50 \mathrm{kOe}$. As expected from $\mathrm{Eq}(1), \Delta S_{\mathrm{m}}$ was maximized, denoted as

$\left|\Delta S_{\max }\right|$, near $T_{\mathrm{C}}$ and increased with $\Delta H$. Additionaly, $\left|\Delta S_{\max }\right|$ point shifts gradually towards high temperature with increasing $\Delta H$. The $\left|\Delta \mathrm{S}_{\max }\right|$ value measured at $\Delta H=50 \mathrm{kOe}$ was the largest which is 
$6.0 \mathrm{~J} / \mathrm{kg} . \mathrm{K}$ at $T \approx 270 \mathrm{~K}$. The $\left|\Delta \mathrm{S}_{\max }\right|$ value measured at $\Delta H=50 \mathrm{kOe}$ for this sample is comparable to those of $\mathrm{La}_{0.7} \mathrm{Ca}_{0.2} \mathrm{Ba}_{0.1} \mathrm{MnO}_{3}[15], \mathrm{La}_{0.87} \mathrm{Sr}_{0.13} \mathrm{MnO}_{3}$ [21], and $\mathrm{La}_{0.7} \mathrm{Sr}_{0.3} \mathrm{Mn}_{0.9} \mathrm{Cu}_{0.1} \mathrm{O}_{3}$ [22].

In addition to the assessment based on $\Delta \mathrm{S}_{\mathrm{m}}$, in order to evaluate the MCE, the other parameter could be considered that is relative cooling power (RCP). This parameter can be calculated according to the magnitude of $\Delta \mathrm{S}_{\mathrm{m}}$ and its full width at half maximum, as

$\mathrm{RCP}=|\Delta \operatorname{Smax}| \times$ FWHM that is shown in [4]. Figure 3(b) shows the dependence of RCP on applied field $\mathrm{H}$. Clearly, the RCP increased with $H$ and under applied field of $\Delta \mathrm{H}=50 \mathrm{kOe}$, its value is $250 \mathrm{~J} / \mathrm{kg}$. This value is large than those of $\mathrm{La}_{0.67} \mathrm{Sr}_{0.33} \mathrm{Mn}_{0.9} M_{0.1} \mathrm{O}_{3}$ with $M=\mathrm{Cr}$, Sn, Ti [23] and $\mathrm{La}_{0.67} \mathrm{Ba}_{0.33} \mathrm{MnO}_{3}[24]$.
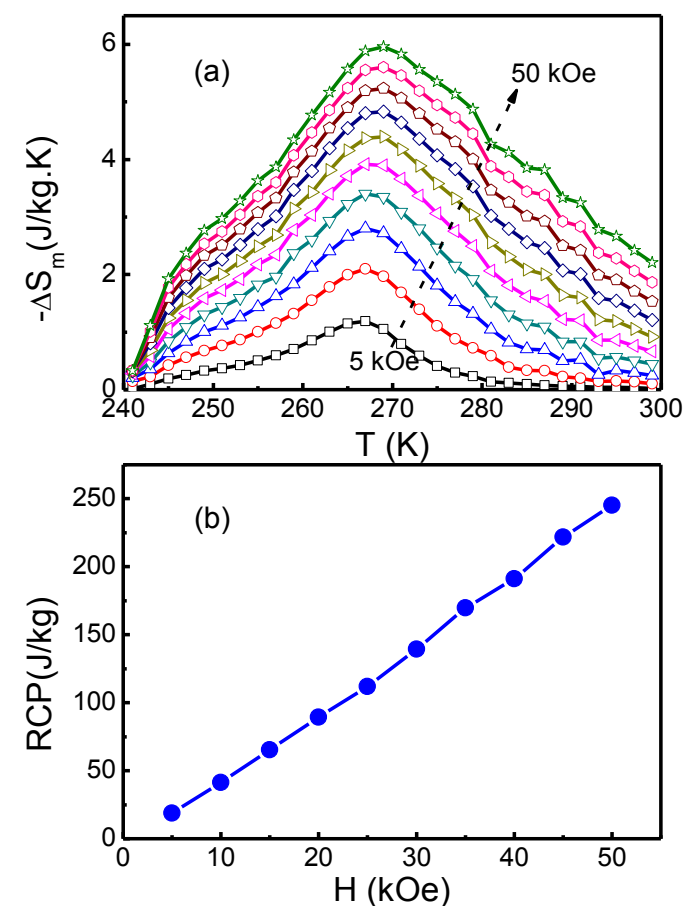

Figure 3. $-\Delta S_{m}(T, H)$ and RCP curves at various $\Delta H$ values for $\mathrm{Nd}_{0.6} \mathrm{Sr}_{0.4} \mathrm{MnO}_{3}$.

Within the framework of second order phase, the field dependence of the magnetic entropy change follows a power law of the filed $\Delta \mathrm{S}_{\mathrm{m}} \alpha \mathrm{H}^{\mathrm{n}}$ with an exponent, $n$, which depends on temperature and field. It can be locally calculated as

$$
\mathrm{n}=\frac{d \ln |\Delta S m|}{d \ln H}
$$

For temperature well below $T_{\mathrm{C}}, \mathrm{n}=1$; well above $T_{\mathrm{C}}, \mathrm{n}=2$ and at $T_{\mathrm{C}}, \mathrm{n}=2 / 3[25,26]$.

Figure 4(a) shows experimental data of $n(T, H)$ which calculated from the $-\Delta S_{\mathrm{m}}(T, H)$. Its minimum $n$ value at $T_{\mathrm{C}}$ is changed from 0.8 (for $\Delta H=5 \mathrm{kOe}$ ) to 0.55 (for $\Delta H=50 \mathrm{kOe}$ ), which are much different from the mean-field-theory value $n=2 / 3$. This can be due to the absence of long-range magnetic order and the existence of magnetic inhomogeneities in this sample.

Recently, Franco et al.[26] have proposed a new criterion based on the entropy change curves to distinguish the order of magnetic transition by means of magnetic measurements. According to them, for ferromagnets undergoing SOMT, the universal curve consists in the collapse $\Delta S_{\mathrm{m}}(T)$ curves 
measured with different maximum applied magnetic fields after a scaling process. Therefore, it is natural to predict a breakdown of the universal curve for ferromagnets undergoing FOMT. The universal curve could be done by normalize all the $\Delta S_{\mathrm{m}}(\mathrm{T})$ curves with their respective peak entropy change, $\Delta S^{\prime}\left(T, H_{\max }\right)$ $=\Delta S_{\mathrm{m}}\left(T, H_{\max }\right) / \Delta S_{\text {peak }}\left(H_{\max }\right)$ and then rescaling the temperature axis defining a new variable $\Theta$,

$$
\theta=\left\{\begin{array}{l}
-(T-T c) /(\operatorname{Tr} 1-T c) \text { for } T \leq T c \\
(T-T c) /(\operatorname{Tr} 2-T c) \text { for } T>T c
\end{array}\right.
$$

The two reference temperatures $T_{\mathrm{r} 1}$ and $T_{\mathrm{r} 2}$ satisfy $T_{\mathrm{r} 1}<T_{\mathrm{C}}<T_{\mathrm{r} 2}$. According to previous report, by using two reference temperatures, it can be avoided the effect of a minority magnetic phase and the demagnetization factor leading to the breakdown of the $\Delta S_{\mathrm{m}}$ curve collapse. These reference temperatures can be selected for each curve from temperature corresponding to $\Delta S_{\mathrm{m}}\left(T_{\mathrm{r}}, H_{\mathrm{max}}\right) /$ $\Delta S_{\text {peak }}\left(H_{\max }\right)=a(0<\mathrm{a}<1)$. The choice of $a$ does not affect the actual construction of the universal curve. In this study, we choose value of $a$ is 0.5 . Figure 4(b) shows the universal curve constructions for the sample by plotting $\Delta S$ ' versus $\theta$. Clearly, all the experimental points collapse onto the master curve, which is consistent with the characteristics of SOMT. Additionally, the Arrott plot constructions confirm that our sample undergo SOMT. This result demonstrates the usefulness of using the universal curve, besides Banerjee's criterion, in assessing the order of magnetic phase transition of perovskite manganites.
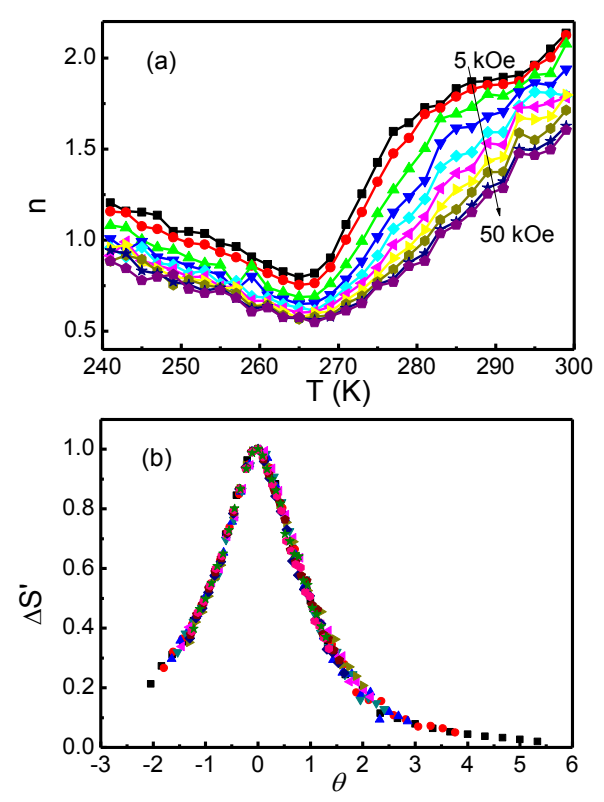

Figure 4. $n(T, H)$ and $-\Delta S^{\prime}$ curves at various $\Delta H$ values for $\mathrm{Nd}_{0.6} \mathrm{Sr}_{0.4} \mathrm{MnO}_{3}$.

\section{Conclusions}

The fabricated ceramic sample $\mathrm{Nd}_{0.6} \mathrm{Sr}_{0.4} \mathrm{MnO}_{3}$ was crystallized in an orthorhombic structure. Detailed analyses of $M(T, H)$ and $-\Delta S_{\mathrm{m}}(T, H)$ data based on the Banerjee's criterion and phenomenological method of constructing the universal entropy curve, demonstrated the existence of second-order magnetic phase transition in this sample. Additionally, we found large MC effect in this sample, particularly $\left|\Delta S_{\max }\right| \approx$ $6.0 \mathrm{~J} / \mathrm{kg} . \mathrm{K}$ and $\mathrm{RCP} \approx 250 \mathrm{~J} / \mathrm{K}$ at $270 \mathrm{~K}$ under an applied field of $50 \mathrm{kOe}$. Such features indicate a potential application of $\mathrm{Nd}_{0.6} \mathrm{Sr}_{0.4} \mathrm{MnO}_{3}$ in magnetic refrigeration close to room temperature. 


\section{Acknowledgements}

This research is funded by Asia Research Center (ARC) under grant number CA.19.05A and is funded by the Domestic Master/PhD Scholarship Programme of Vingroup Innovation Foundation

\section{References}

[1] D.N.H. Nam, R. Mathieu, P. Nordblad, N.V. Khiem, N.X. Phuc, Ferromagnetism and frustration in $\mathrm{Nd}_{0.7} \mathrm{Sr}_{0.3} \mathrm{MnO}_{3}$, Phys. Rev. B 62(2000) 1027-1032. https://doi.org/10.1103/PhysRevB.62.1027.

[2] X. Moya, L.E. Hueso, F. Maccherozzi, A.I. Tovstolytkin, D.I. Podyalovskii, C. Ducati, et al., Giant and reversible extrinsic magnetocaloric effects in $\mathrm{La}_{0.7} \mathrm{Ca}_{0.3} \mathrm{MnO}_{3}$ films due to strain, Nat. Mater. 2(2013) 52-58. https://doi.org/10.1038/nmat3463.

[3] A. Dhahri, F.I.H. Rhouma, S. Mnefgui, J. Dhahri, E.K. Hlil, Room temperature critical behavior and magnetocaloric properties of $\mathrm{La}_{0.6} \mathrm{Nd}_{0.1}(\mathrm{CaSr})_{0.3} \mathrm{Mn}_{0.9} \mathrm{~V}_{0.1} \mathrm{O}_{3}$, Ceram. Int. 40(2014) 459-464. https://doi.org/10.1016/j.ceramint.2013.06.024.

[4] M.-H. Phan, S.-C. Yu, Review of the magnetocaloric effect in manganite materials, J. Magn. Magn. Mater 308(2007) 325-340. https://doi.org/10.1016/j.jmmm.2006.07.025.

[5] C. Zener, Interaction between the d-shells in the transition metals. II. ferromagnetic compounds of manganese with perovskite structure, Phys. Rev. B 82(1951) 403-408. https://doi.org/10.1103/PhysRev.82.403.

[6] A.J. Millis, P.B. Littlewood, B.I. Shraiman, Double exchange alone does not explain the resistivity of La ${ }_{x} \mathrm{Sr}_{\mathrm{x}} \mathrm{MnO}_{3}$, Phys. Rev. Lett. 74(1995) 5144-5147. https://doi.org/10.1103/PhysRevLett.74.5144.

[7] M.B. Salamon, P. Lin, S.H. Chun, Colossal magnetoresistance is a griffiths singularity, Phys. Rev. Lett. 88 (2002) 197203-197206. https://doi.org/10.1103/PhysRevLett.88.197203.

[8] L. Demkó, I. Kézsmárki, G. Mihály, N. Takeshita, Y. Tomioka, and Y. Tokura, Multicritical end point of the firstorder ferromagnetic transition in colossal magnetoresistive manganites, Phys. Rev. Lett. 101(2008) 037206037209. https://doi.org/10.1103/PhysRevLett.101.037206.

[9] A. Munoz, J.A. Alonso, M.J. Martinez-Lope, J.L. Garcia-Munoz, M.T. Fernandez-Diaz, Magnetic structure evolution of $\mathrm{NdMnO}_{3}$ derived from neutron diffraction data, J. Phys.: Condens. Matter 12 (2000) 1361-1368. https://doi.org/10.1088/0953-8984/12/7/319.

[10] R. Venkatesh, M. Pattabiraman, K. Sethupathi, G. Rangarajan, S. Angappane, and J.-G. Park, Tricritical point and magnetocaloric effect of $\mathrm{Nd}_{1-\mathrm{x}} \mathrm{Sr}_{\mathrm{x}} \mathrm{MnO}_{3}$, J. Appl. Phys. 103(2008) 07B319-07B322. https://doi.org/10.1063/1.2832412.

[11] R. Venkatesh, M. Pattabiraman, S. Angappane, G. Rangarajan, K. Sethupathi, J. Karatha, Complex ferromagnetic state and magnetocaloric effect in single crystalline $\mathrm{Nd}_{0.7} \mathrm{Sr}_{0.3} \mathrm{MnO}_{3}$, Phys. Rev. B 75 (2007) 224415-224418. https://doi.org/10.1103/PhysRevB.75.224415.

[12] R.S. Freitas, L. Ghivelder, F. Damay, F. Dias, L.F. Cohen, Magnetic relaxation phenomena and cluster glass properties of $\mathrm{La}_{0.7-\mathrm{x}} \mathrm{Y}_{\mathrm{x}} \mathrm{Ca}_{0.3} \mathrm{MnO}_{3}$ manganites, Phys. Rev. B 64 (2001) 144404-144407. https://doi.org/10.1103/PhysRevB.64.144404.

[13] T.A. Ho, S.H. Lim, C.M. Kim, M.H. Jung, T.O. Ho, P.T. Tho, Magnetic and magnetocaloric properties of La $\mathrm{La}_{0.6} \mathrm{Ca}_{0.4-}$ ${ }_{x} \mathrm{Ce}_{\mathrm{x}} \mathrm{MnO}_{3}$, J. Magn. Magn. Mater 438(2017) 52-59. https://doi.org/10.1016/j.jmmm.2017.04.038.

[14] T.A. Ho, S.H. Lim, P.T. Tho, T.L. Phan, S.C. Yu, Magnetic and magnetocaloric properties of $\mathrm{La}_{0.7} \mathrm{Ca}_{0.3} \mathrm{Mn}_{1-\mathrm{x}} \mathrm{Zn}_{\mathrm{x}} \mathrm{O}_{3}$, J. Magn. Magn. Mater 426(2017) 18-24. https://doi.org/10.1016/j.jmmm.2016.11.050.

[15] T.-L. Phan, N.T. Dang, T.A. Ho, T.V. Manh, T.D. Thanh, C.U. Jung, First to second order magnetic phase transition $\mathrm{La}_{0.7} \mathrm{Ca}_{0.3-\mathrm{x}} \mathrm{Ba}_{\mathrm{x}} \mathrm{MnO}_{3}$ exhibiting large magnetocaloric effect, J. Alloys Compd. 657(2016) 818-834. https://doi.org/10.1016/j.jallcom.2015.10.162.

[16] T.A. Ho, D.-T. Quach, T.D. Thanh, T.O. Ho, M.H. Phan, T.L. Phan, Magnetocaloric effect and critical behavior in a disordered ferromagnet $\mathrm{La}_{0.7} \mathrm{Sr}_{0.3} \mathrm{Mn}_{0.9} \mathrm{Ti}_{0.1} \mathrm{O}_{3}$, IEEE Trans. Magn. 51(2015) 2501304-2501307. https://doi.org/10.1109/TMAG.2015.2436383. 
[17] A. Arrott., Criterion for ferromagnetism from observations of magnetic isotherms, Phys. Rev. 108(1957) 13941397. https://doi.org/10.1103/PhysRev.108.1394.

[18] S.K. Banerjee, On a generalised approach to first and second order magnetic transitions, Phys. Lett.12 (1964) 1622. https://doi.org/10.1016/0031-9163(64)91158-8.

[19] V. Franco, J. S. Blázquez, A. Conde, Field dependence of the magnetocaloric effect in materials with a second order phase transition: A master curve for the magnetic entropy change, Appl. Phys. Lett. 89 (2006) 222512222517. https://doi.org/10.1063/1.2399361.

[20] N.S. Bingham, M.H. Phan, H. Srikanth, T.M.A., C. Leighton, Magnetocaloric effect and refrigerant capacity in charge-ordered manganites, J. Appl. Phys. 106(2009) 023909-023914. https://doi.org/10.1063/1.3174396.

[21] A. Szewczyk, H. Szymczak, A. Wisniewski, K. Piotrowski, R. Kartaszynski, B. Dabrowski, et al., Magnetocaloric effect in $\mathrm{La}_{1-\mathrm{x}} \mathrm{Sr}_{\mathrm{x}} \mathrm{MnO}_{3}$ for $\mathrm{x}=0.13$ and 0.16, Appl. Phys. Lett. 77(2000) 1026-1029. https://doi.org/10.1063/1.1288671.

[22] M.H. Phan, H.X. Peng, S.C. Yu, N. Tho, N. Chau, Large magnetic entropy change in Cu-doped manganites, J. Magn. Magn. Mater. 285(2005) 199-203. https://doi.org/10.1016/j.jmmm.2004.07.041.

[23] B. Arayedh, S.Kallel, N. Kallel, O.Pena, Influence of non-magnetic and magnetic ions on the magnetocaloric properties of $\mathrm{La}_{0.7} \mathrm{Sr}_{0.3} \mathrm{Mn}_{0.9} \mathrm{M}_{0.1} \mathrm{O}_{3}$, J. Magn. Magn. Mater 361(2014) 68-73. https://doi.org/10.1016/j.jmmm.2014.02.075.

[24] D.T. Morelli, A.M. Mance, J.V. Mantese, A.L. Micheli, Magnetocaloric properties of doped lanthanum manganite films, J. Appl. Phys. 79 (1996) 373-376. https://doi.org/10.1063/1.360840.

[25] V. Franco, A. Conde, Scaling laws for the magnetocaloric effect in second order phase transitions: From physics to applications for the characterization of materials, Int. J. Refri. 33(2010) 465-473. https://doi.org/10.1016/j.ijrefrig.2009.12.019.

[26] V. Franco, J.S. Blazquez., A. Conde, Field dependence of the magnetocaloric effect in materials with a second order phase transition: A master curve for the magnetic entropy change, Appl. Phys. Lett. 89(2006) 222512-222515. https://doi.org/10.1063/1.2399361. 